\title{
Strategies for Early Identification of Atherosclerosis in Systemic Autoimmune Disease
}

Turiel $\mathbf{M}^{1^{*}}$, Gianturco $L^{1}$, Galaverna $\mathbf{S}^{1}$, Colombo $\mathbf{C}^{1}$, Stella $\mathbf{D}^{1}$, Sarzi-Puttini $\mathbf{P}^{2}$, Atzeni $\mathrm{F}^{2}$ and Bodini BD

${ }^{1}$ IRCCS Galeazzi Orthopedic Institute, Cardiology Unit, Milan, Italy

${ }^{2}$ L. Sacco University Hospital, Rheumatology Unit, Milan, Italy

3IRCCS Galeazzi Orthopedic Institute, Rehabilitation Unit, Milan, Italy

Received date: May 29, 2014, Accepted date: July 18, 2014, Published date: July 25, 2014

*Corresponding author: Maurizio Turiel, Director of Cardiology Unit, IRCCS Galeazzi Orthopaedic Institute, via R. Galeazzi, 420161 Milan, Italy, Tel: +390250319955; Fax: +390250319956; E-mail: maurizio.turiel@unimi.it

Copyright: (c) 2014 Maurizio Turiel, et al. This is an open-access article distributed under the terms of the Creative Commons Attribution License, which permits unrestricted use, distribution, and reproduction in any medium, provided the original author and source are credited.

\begin{abstract}
Systemic autoimmune diseases (SADs) are associated with significantly enhanced cardiovascular (CV) morbidity and mortality due to a cluster of risk factors. Among them we find traditional markers of CV risk but also specific risk factors principally related to inflammation and autoimmunity. Therefore, CV involvement assessment in those diseases is more and more important and several authors have been studying for the last years that phenomenon.

The most important goal for all of them is CV prevention and follow-up of subjects with such abnormalities; in particular, CV burden is mainly due to atherosclerosis (ATS).

So, in order to achieve the best CV prevention program a very early diagnose of ATS in these patients (pts) is fundamental, especially in those phases of disease in which no symptoms are present and clinical manifestations are not clearly visible.

In this review, our aim was to find the best marker for identifying early ATS in systemic autoimmune diseases (SADs) by starting our long experience in this field.
\end{abstract}

Keywords: Atherosclerosis; Systemic autoimmune diseases; ADMA

\section{Introduction}

Systemic autoimmune diseases (SADs) are quite widespread syndromes with an incidence of 0.13-0.16 in general population [1]. They are characterized by an important inflammatory response associated with a much or less large grade of autoimmunity mechanisms [2].

Generally, they consist of a multi-organ involvement including cardiovascular (CV) system [3,4]. Therefore, among systemic autoimmune diseases (SADs) patients (pts) we can include those with rheumatoid arthritis (RA), systemic sclerosis (SSc), psoriatic arthritis (PsA), Sjogren's syndrome (SS) and other minor connective tissue diseases (CTDs).

For long years in literature several manuscripts about this topic have been published and nowadays we know that CV involvement plays a leading role in genesis of morbidity and mortality of these pts [5]. Moreover, CV disease (CVD) is very often asymptomatic especially in early stages [6]. Thus, it is pivotal to reach an early diagnose in these subjects in order to organize an opportune follow-up and a winning strategy of prevention. Early identification of $\mathrm{CV}$ damage is possible and it is very very important. In fact, we know that principal actor of CV manifestations is atherosclerosis (ATS) but we also know primum movens of atherosclerotic lesions is represented by endothelial dysfunction that is a reversible process. So, we know that pathogenesis of ATS in SADs is not completely clear yet, but however it is acquainted that endothelial dysfunction is the first stage of whole process [7]. And it is once more actual that our aim is battle against endothelial injury in order to slow down ATS. Probably, we can't stop genesis of atherosclerotic plaque but we would like to retard its formation. Thus, we have to take part on endothelium to prevent its dysfunction and/or to control related complications. We may imagine our important interference in the travel that brings us from endothelial dysfunction to ATS towards inflammation [8]. Here it is our goal, our hopefulness: we would like to manage ATS more and more better in the future.

Along the years our group have investigated a large cohort of pts with SADs analysing different items by means of several techniques, first of all, non-invasive assessment of coronary flow reserve (CFR) with transthoracic echocardiography. Then, we have optimised our studies using aymmetric dymethilarginine (ADMA) and also other more recent indexes of $\mathrm{CV}$ damage.

So, nowhere to obtain the goal of early diagnose we have several instruments and validated methods and so it is not difficult to do that, but is mandatory routinary use of these tools in selected populations. In a very recent Hungarian polycentric study a meticulous list of these tools has carried out [9]. So, we have only to use them in the most opportune way with the most adequate choose in relation to specific situation, on the basis of our personal experience and especially related to international scientific data emerging from literature.

Besides that, if we consider endothelium as the principal target of our prevention program we have to focus our attention on ADMA that 
is one of the most specific markers of $\mathrm{CV}$ risk related to endothelial function [10].

Finally, it is increasingly desirable to achieve better results the formation of teams working among cardiologists, rheumatologists and other specialists involved in care and follow-up of these pts.

\section{Strategies for Identifying Atherosclerosis (Ats)}

Along the years we have been studying a large number of pts with CTDs and SADs. For all of them we have data resulting from the following analysis: CFR values, ADMA plasma dosages and finally common carotid intima-media thickness (cIMT) evaluation.

In minimal percentage we have also studied other specific items but not all of them and not always were present.

The known frequency of CV involvement in SRDs is greatly affected by the diagnostic methods used, and recent imaging techniques can detect subtle preclinical abnormalities, although their clinical significance has not yet been established [11].

Traditionally, cIMT measurement was used to assess ATS grading $[12,13]$. But anatomical changes that can be observed by means of carotid ultrasound studies are a later marker of CV injury.

Moreover, the evaluation of coronary flow reserve (CFR) by means of transthoracic dipyridamole stress echocardiography has proved to be a highly sensitive $(>90 \%)$ diagnostic marker of CAD $[14,15]$ and, when associated with regional wall motion analysis, is also highly specific [16]. It has been shown that a CFR $<2$ assessed in the distal left anterior descending artery (LAD) accurately predicts the presence of coronary stenosis and, in the absence of epicardial coronary stenosis, an abnormal CFR may reflect an impaired coronary microcirculation as found in various clinical settings characterised by a high CV risk [17]. The evaluation of CFR also has prognostic value, as a reduced CFR correlates with a negative prognosis [18]. With the pts in a stable $90^{\circ}$ left lateral recumbent position, and using a modified two-chamber view to identify distal the LAD, LAD CFR can be evaluated before and during dipyridamole infusion $(0.84 \mathrm{mg} / \mathrm{kg}$ over 6 minutes) using an high-frequency transducer to assess the systolic and diastolic components of the Doppler signal. Coronary blood flow in the middistal portion of the LAD is measured under the guidance of colourDoppler flow mapping by synchronising the electrocardiogram, and CFR is calculated as the ratio between peak diastolic velocity during hyperemia and diastolic velocity at baseline (Figure 1).

At the end of the analysis $125-250 \mathrm{mg}$ of aminophylline should be administered to counteract the effect of dipyridamole. Hirata et al. [19] used stress echocardiography to evaluate CFR in premenopausal women with systemic lupus erythematosus (SLE), and found that it was significantly reduced in comparison with age and gender-matched controls. They concluded that microvascular impairment in SLE could be explained by a functional alteration in the endothelium, which is responsible for the decreased vasodilatation in response to pharmacological stress. At the same time, Turiel et al. first studied pts with longstanding rheumatoid arthritis (RA) and subsequently pts with early RA and no sign or symptom of coronary artery disease who were off-therapy with steroids and/or anti-rheumatic drugs, and observed a reduction in CFR between the latter and healthy controls $(2.5 \pm 0.6$ vs. $3.5 \pm 0.8, \mathrm{p}<0.01)[20,21]$.

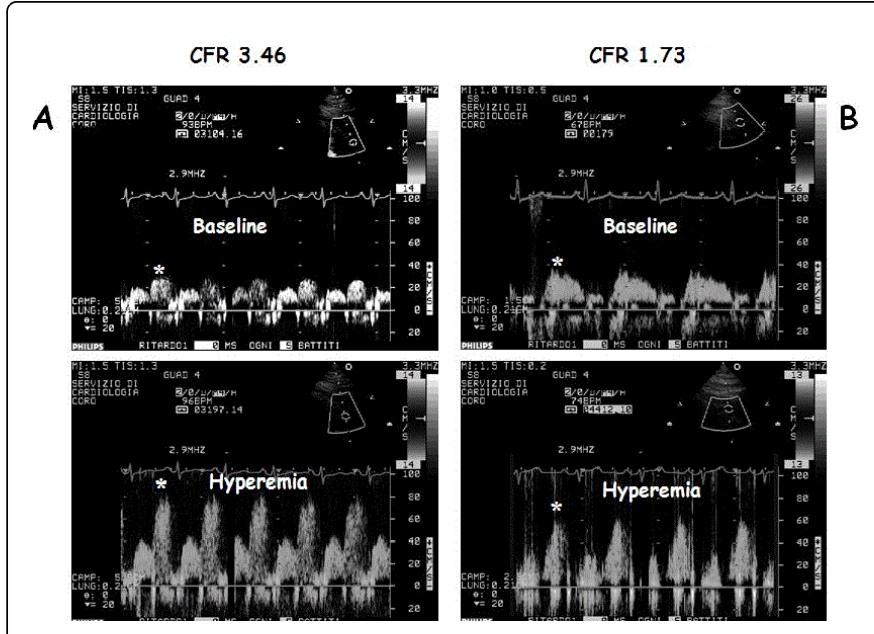

Figure 1: Pulse-wave (PW) Doppler sampling of LAD with evaluation of CFR. A) Example in a healthy subject, respectively at baseline (upper image) and after i.v. dipyridamole administration (lower image) inducing hyperaemia. B) Example in a SADs outpatient.

In particular, $48 \%$ of the early RA pts had a CFR of $>2.5,28 \%$ a CFR of $2-2.5$, and $24 \%$ a CFR of $\leq 2$ consistent with severe epicardial stenosis. Moreover, in addition to reducing disease activity and inflammatory status, 18 months' antirheumatic therapy led to a significant improvement in CFR in the early RA pts [22]. Tissue Doppler imaging (TDI) and speckle tracking echocardiography (STE) have recently been introduced in order to overcome the limitations of standard echocardiography in assessing myocardial function. In particular, TDI has been considered a reliable means of detecting subclinical cardiac involvement in systemic sclerosis pts with normal standard echo findings, and Birdane et al. have shown that RA pts have significantly impaired left and right ventricular TDI parameters in comparison with healthy controls $[23,24]$. However, the method is limited by its angle dependency because only deformations along the ultrasound beam can be derived from velocities, whereas the myocardium simultaneously deforms in three dimensions. Furthermore, TDI cannot differentiate active and passive myocardial motion, which requires high frame rate imaging [25]. STE is emerging as an objective and reproducible means of quantifying global and regional myocardial function on the basis of echocardiographic images, and its sensitivity makes it reliable in detecting early silent cardiac involvement in various clinical settings, including connective tissue diseases [26].

It has recently been demonstrated that endothelial dysfunction represents an early stage of atherogenesis in young pts with long-term RA and low disease activity [27].

Our group have recently observed an inverse correlation between plasma ADMA levels and CFR in early RA pts without CAD, which indicates that there is a relationship between endothelial dysfunction and coronary microvascular impairment. In conclusion, as CV disease is the main cause of mortality and morbidity in pts with SRDs, it is essential to detect endothelial dysfunction and impaired coronary microcirculation in asymptomatic subjects as soon as possible [21].

Thus, echocardiographic assessments (CFR evaluation, TDI and STE) currently seem to be the most suitable means of screening and 
Page 3 of 4

diagnosis, and new biochemical markers such as plasma ADMA levels could be used as a simple way of screening SADs pts at higher CV risk who need more aggressive treatment capable of slowing or reversing the progression of atherosclerosis [25].

In conclusion, about ADMA, we can tell that is the main endogenous inhibitor of all three NO synthases, thus causing endothelial dysfunction and promoting the progression of atherosclerosis. In one of the most important our studies we showed that plasma ADMA levels were significantly higher in the early RA pts suggesting an early atherosclerosis stage in this population [21].

Instead, new tools analyzing stiffness capabilities are not so diffuse in clinical practice and further investigations will be necessary in order to adequately valorising them [28].

\section{Results}

Here they are our findings in different types of rheumatic diseases. We have recently conducted a post-hoc re-calculation of our data.

Analyzing the markers used for ATS evaluation we can observe that ADMA values are always very important as indicator of ATS and/or endothelial dysfunction in the large spectrum of SADs: RA, PsA, SSc and SS. In fact, in all our retrospective studies the Odds Ratio (OR) analysis has obtained significative results. More in details, in comparison with controls' groups of healthy subjects, we have ADMA plasma values above the normal ranges in $\mathrm{RA}(\mathrm{OR}=3.89$; 95\% CI 1.99-5.69), then in PsA (OR=2.49; 95\% CI 1.16-3.21), in SSc (OR=1.96; $95 \%$ CI 1.08-2.31) and finally in SS (1.13; 95\% CI 0.99-2.06) (Table 1).

\begin{tabular}{|l|l|l|l|l|}
\hline Parameters & RA (OR) 95\% Cl & PSA (OR) 95\% Cl & SSc (OR) 95\% Cl & SS (OR) 95\% Cl \\
\hline \multirow{3}{*}{ ADMA } & 3.89 & 2.49 & 1.96 & 1.13 \\
\cline { 2 - 6 } & $1.99-5.69$ & $1.16-3.21$ & $1.08-2.31$ & $0.99-2.06$ \\
\hline \multirow{2}{*}{ CFR } & 1.88 & 1.76 & 1.02 & 0.95 \\
\cline { 2 - 6 } & $0.96-2.22$ & $0.89-2.00$ & $0.78-1.93$ & $0.68-1.05$ \\
\hline \multirow{2}{*}{ CIMT } & 0.93 & 0.95 & 0.88 & 0.89 \\
\cline { 2 - 5 } & $0.65-1.23$ & $0.73-2.02$ & $0.62-1.08$ & $0.56-1.45$ \\
\hline
\end{tabular}

Table 1: Results Chart. OR: odds ratio; 95\% CI= 95\% confidential interval.

On the contrary, another very important index such as CFR had the profile shown in the results' chart, as like as cIMT.

And so, we can observe a different "weight" of ADMA and CFR in discovering ATS abnormalities in SADs. But, we will give you more details about these findings in the following discussion/conclusions paragraph.

\section{Discussion and Conclusions}

In our studies ADMA was one of the most important markers for evaluation of endothelial dysfunction.

We may better interpret data about ADMA "weight" in identifying ATS if we consider characteristics of our populations in study. Such pts usually were in early phases of disease and so only an accurate index as ADMA able to investigate preclinical endothelial dysfunction could point out subclinical abnormalities.

We also used other instruments such as CFR, cIMT and finally stiffness parameters, but no one were fundamental like ADMA.

In particular, about new insights known as stiffness indexes, in literature at the moment, we have a bit volume of data and a unified and clear interpretation is not possible yet. Therefore, nowhere, we may not consider them as reliable tools.

If we re-analyze our publication, above mentioned, in a large number of SADs, we can find in all of them an early involvement of the endogen molecule known as ADMA. This one is responsible of first step of ATS better known as endothelial dysfunction that is a potentially reversible process.
Our findings in fact show a statistical and significantly difference for ADMA in pts with RA, PsA, SSc and SS in comparison with our control groups.

The difference appreciated in our wide case histories allows us to underline that ADMA can be considered nowadays as one of the best marker of endothelial dysfunction even in consideration of its relative low-cost of using and because of it is a very simple and suitable tool perceived as comfortable by pts.

For the future, we can hypothesize a most important role of ADMA and progressively also of other new indexes in screening of SADs about CV system.

In particular, the so-called " $\mathrm{CV}$ coupling" resulting of heart analysis and vascular assessment will be the new frontier for researchers working in cardio-rheumatology field. And besides that, we should wish an introduction of a cluster of markers for identifying ATS with more and more possibility to grow in our knowledge, prevention programs, but especially, in care of pts and related outcomes.

Obviously, the most important study limitation is related to source of data: all of findings are collected in our Cardiology Unit and a multi-centric approach should be desirable with all troubles related to organization of such studies.

\section{References}

1. Peschken CA, Hitchon C (2012) Rising prevalence of systemic autoimmune rheumatic disease: increased awareness, increased disease or increased survival? Arthritis Research \& Therapy 14: A20.

2. Doria A, Putterman C, Sarzi-Puttini P, Szekanecz Z, Shoenfeld Y (2012) Controversies in rheumatism and autoimmunity. Autoimmun Rev 11: 555-557. 
Citation: Turiel M, Gianturco L, Galaverna S, Colombo C, Stella D, et al. (2014) Strategies for Early Identification Of Atherosclerosis in Systemic Autoimmune Disease. J Cardiovasc Dis Diagn 2: 167. doi:10.4172/2329-9517.1000167

Page 4 of 4

3. Hollan I, Meroni PL, Ahearn JM, Cohen Tervaert JW, Curran S, et al (2013) Cardiovascular disease in autoimmune rheumatic diseases. Autoimmun Rev 12: 1004-1015.

4. Gaetano Santulli (2013) Epidemiology of Cardiovascular Disease in the 21st Century: Updated Numbers and Updated Facts. JCvD 1:1-2.

5. Villa-Forte A, Mandell BF (2011) Cardiovascular disorders and rheumatic disease. Rev Esp Cardiol 64: 809-817.

6. Turiel M, Sarzi-Puttini P, Atzeni F, De Gennaro Colonna V, Gianturco L, et al. (2011) Cardiovascular injury in systemic autoimmune diseases: an update. Intern Emerg Med 6 Suppl 1: 99-102.

7. Murdaca G, Colombo BM, Cagnati P, Gulli R, Spanò F, et al. (2012) Endothelial dysfunction in rheumatic autoimmune diseases. Atherosclerosis 224: 309-317.

8. Dimitroulas T, Sandoo A, Kitas GD (2012) Asymmetric dimethylarginine as a surrogate marker of endothelial dysfunction and cardiovascular risk in patients with systemic rheumatic diseases. Int J Mol Sci 13: 12315-12335.

9. Kerekes G, Soltész P, Nurmohamed MT, Gonzalez-Gay MA, Turiel M, et al. (2012) Validated methods for assessment of subclinical atherosclerosis in rheumatology. Nat Rev Rheumatol 8: 224-234.

10. Chen XM, Hu CP, Li YJ, Jiang JL (2012) Cardiovascular risk in autoimmune disorders: role of asymmetric dimethylarginine. Eur J Pharmacol 696: 5-11.

11. Recio-Mayoral A, Mason JC, Kaski JC, Rubens MB, Harari OA, et al (2009) Chronic inflammation and coronary microvascular dysfunction in patients without risk factors for coronary artery disease. Eur Heart J 30: 1837-1843.

12. Butt MU, Zakaria M (2009) Association of common carotid intimal medial thickness (CCA-IMT) with risk factors of atherosclerosis in patients with type 2 diabetes mellitus. J Pak Med Assoc 59: 590-593.

13. Thakore AH, Guo CY, Larson MG, Corey D, Wang TJ, et al. (2007) Association of multiple inflammatory markers with carotid intimal medial thickness and stenosis (from the Framingham Heart Study). Am J Cardiol 99: 1598-1602.

14. Caiati C, Zedda N, Montaldo C, Montisci R, Iliceto S (1999) Contrastenhanced transthoracic second harmonic echo Doppler with adenosine: noninvasive, rapid and effective method for coronary flow reserve assessment. J Am Coll Cardiol 34: 122-130.

15. Hozumi T, Yoshida K, Ogata Y, Akasaka T, Asami Y (1998) Noninvasive assessment of significant left anterior descending coronary artery stenosis by coronary flow velocity reserve with transthoracic color Doppler echocardiography. Circulation 97: 1557-1562.
16. Rigo F, Richieri M, Pasanisi E, Cutaia V, Zanella C, et al. (2003) Usefulness of coronary flow reserve over regional wall motion when added to dual-imaging dipyridamole echocardiography. Am J Cardiol 91: 269-273.

17. Dimitrow PP (2002) Coronary flow reserve-measurement and application: focus on transthoracic Doppler echocardiography, Kluwer Academic Publishers, London.

18. Rigo F, Gherardi S, Galderisi M, Pratali L, Cortigiani L, et al. (2006) The prognostic impact of coronary flow-reserve assessed by Doppler echocardiography in non-ischaemic dilated cardiomyopathy. Eur Heart J 27: 1319-1323.

19. Hirata K, Kadirvelu A, Kinjo M, Sciacca R, Sugioka K, et al. (2007) Altered coronary vasomotor function in young patients with systemic lupus erythematosus. Arthritis Rheum 56: 1904-1909.

20. Turiel M, Atzeni F, Tomasoni L, de Portu S, Delfino L, et al. (2009) Noninvasive assessment of coronary flow reserve and ADMA levels: a casecontrol study of early rheumatoid arthritis patients. Rheumatology (Oxford) 48: 834-839.

21. Atzeni F, Sarzi-Puttini P, De Blasio G, Delfino L, Tomasoni L, et al (2007) Preclinical impairment of coronary flow reserve in patients with rheumatoid arthritis. Ann N Y Acad Sci 1108: 392-397.

22. Turiel M, Tomasoni L, Sitia S, Cicala S, Gianturco L, et al. (2010) Effects of long-term disease-modifying antirheumatic drugs on endothelial function in patients with early rheumatoid arthritis. Cardiovasc Ther 28: e53-64.

23. Turiel M, Peretti R, Mornati D, Delfino L, Atzeni F, et al. (2005) The role of echocardiographic techniques in connective tissue diseases. Autoimmun Rev 4: 171-177.

24. Birdane A, Korkmaz C, Ata N, Cavusoglu Y, Kasifoglu T, et al. (2007) Tissue Doppler imaging in the evaluation of the left and right ventricular diastolic functions in rheumatoid arthritis. Echocardiography 24: 485-493.

25. Nesbitt GC, Mankad S, Oh JK (2009) Strain imaging in echocardiography: methods and clinical applications. Int J Cardiovasc Imaging 25 Suppl 1: 9-22.

26. Sitia S, Gianturco L, Tomasoni L, Turiel M (2010) Role of cardiovascular imaging in systemic autoimmune diseases. World J Cardiol 2: 237-242.

27. Hänsel S, Lässig G, Pistrosch F, Passauer J (2003) Endothelial dysfunction in young patients with long-term rheumatoid arthritis and low disease activity. Atherosclerosis 170: 177-180.

28. Yildiz M (2010) Arterial distensibility in chronic inflammatory rheumatic disorders. Open Cardiovasc Med J 4: 83-88. 